\title{
NOISE REDUCED HIGH DYNAMIC RANGE TONE MAPPING USING INFORMATION CONTENT WEIGHTS
}

Zijian Zhu, Zhengguo Li, Shiqian Wu

Institute for Infocomm Research, Singapore
Pasi Fränti

University of Eastern Finland, Finland

\begin{abstract}
In this paper, we propose a noise reduced tone mapping method based on information content weights, where the perceptually unimportant pixels are smoothed during the decomposition in two steps. First, a saliency-based information content weight is introduced to give high fidelity to the data term based on the ratio of the local pixel power and the overall noise power in the base layer decomposition. Then, the detail layer is subtracted using the mutual informationbased information content weight from the original image luminance and the clean base layer. Experiments show the effectiveness of the proposed method in the improvements of both signal-to-noise ratio and visual quality.
\end{abstract}

Index Terms - high dynamic range, tone mapping, edgepreserving decomposition, de-noising, information content

\section{INTRODUCTION}

Dynamic range of a real world scene is defined as the ratio between the largest and the smallest light intensities in the scene. Due to hardware limitation, an image captured using conventional camera is not enough to keep the full dynamic range. Therefore, a high dynamic range (HDR) image is usually reconstructed using either newly designed sensors [1] or synthesized using multiple differently exposed images [2]. Unfortunately, an HDR image cannot be displayed directly on a conventional display device due to hardware limitations. Although HDR-solution-based monitor [3] and projector [4] have been proposed, they are not widely used due to quality and cost issues. Thus, compression from an HDR image into a display-able image is studied as HDR tone mapping, and global operators [5,6] and local operators [7-9] have been proposed.

Most of these algorithms only focused on how to keep fine details, but did not consider noise. The noise in an HDR image, especially for a low lighting HDR scene, is inherited from the capturing device. A large ISO setting is usually used at low lighting conditions which results in a noisy HDR image. Unfortunately, it can be easily treated as the fine detail and retained in the final image. In particular, the low frequency coarse-grain noise is always mixed with small details, which, in some tone mapping operators, is even enhanced in the final output.

The gradient-decomposition-based method [10] magnifies the small magnitude to review the fine details. Noise, if not carefully treated, can be magnified and become more obvious in the tone mapping result. In the edge-preservingbased method [7,9], both fine details and coarse-grain noise are retained in the detail layer. In the scale-decompositionbased method [11], high frequency band which contains large edges and high frequency noise are compressed and reduced. However, the low-frequency bands with coarse-grain noise are retained.

In order to reduce noise, Lee et al. proposed a scaledecomposition-based method [12]. It used a discrete Haar wavelet transform to decompose an HDR image into four subbands. A noise reduction step was introduced by filtering the subband with the lowest frequency using bilateral filter, and smoothing the rest subbands using soft-thresholding. The problem of using multiscale techniques is that the original signal may be distorted at the composition stage which generates the halo artifacts [11], if the parameters are not carefully selected.

In this paper, a noise reduced tone mapping algorithm is proposed by using two information content weights (ICW). These ICWs are incorporated in an edge-preserving tone mapping method for fast and effective processing. The first ICW is a saliency map that represents the importance of the received information by treating the original HDR image as a clean image passes through a noisy channel. It is used to generate a clean base layer by controlling the fidelity between the base layer and the original HDR image. The second ICW is defined as mutual information between a noise image and a clean reference image using a simplified information fidelity criterion [13]. It is used in the detail layer substraction by regarding the base layer as a clean reference. Experiments show that the proposed method can reduce the noise effectively.

The rest of the paper is organized as follows. Section II describes the proposed ICWs and the noise reduced tone mapping process. Experimental results, comparison and discussion are provided in Section III. And the paper is concluded in Section IV. 


\section{NOISE REDUCED TONE MAPPING}

In the Retinex theory [14], an image $(I)$ is regarded as a product of two components: an illuminance component which contains large luminance variance, and a reflectance component which contains intrinsic information. Base on this, an HDR image is decomposed into a base layer $(B)$ with large luminance variance and a detail layer $(D)$ with fine details. Here, $B, D$ and $I$ are all defined in log luminance domain, and therefore, the original product is rewrite as $I=B+D$. The proposed ICWs work on the base layer and the detail layer respectively.

\subsection{Saliency-based Decomposition}

A noise image can be regarded as a clean image that passes through a noisy visual channel. Thus, the local information content of this noise image is quantified as the number of bits that can be received from the noisy visual channel [15]. Inspired by information theory on how information is received through a noisy channel, a saliency-based ICW is defined as

$$
S(p)=\frac{1}{2} \log _{2}\left(1+\frac{\sigma^{2}(p)}{\sigma_{c}^{2}}\right),
$$

where $\sigma^{2}(p)$ denotes the local variance at each pixel $p$ with a small window, and $\sigma_{c}^{2}$ is a constant represents the channel noise power.

The base layer is derived using the saliency-based ICW for better data fidelity at more important regions, and smooth the less important regions in the regularization term by seeking the minimum of

$$
\iint\left(S(p) \cdot(B(p)-I(p))^{2}+\lambda \Phi(B(p), I(p))\right) d x d y,
$$

where $\lambda$ is a smoothing coefficient and $\Phi$ represents a regularization term. The principle is that a higher weight is given to pixels that are perceptually more sensitive in assessing the image quality, and therefore, the base layer will be more close to the original image. On the contrary, when processing pixels that are less sensitive in the human perceptual, commonly low frequency components, the decomposition is bias towards the regularization term for smoothing.

In this paper, we use the regularization term from the weight-least-square (WLS) method [7]. The full objective function (2) can then be rewritten using matrix notation as

$$
(b-i)^{T} s(b-i)+\lambda\left(b^{T} D_{x}^{T} A_{x} D_{x} b+b^{T} D_{y}^{T} A_{y} D_{y} b\right),
$$

where $b, i$ and $s$ are the vector representation of $B, I$ and $S$, $A_{x}$ and $A_{y}$ are diagonal matrices containing the smoothness weights, and $D_{x}$ and $D_{y}$ denote discrete differentiation operators. A linear system is derived by minimizing the objective function as

$$
\left(I_{m}+\lambda s^{-1} \Psi\right) b=i,
$$

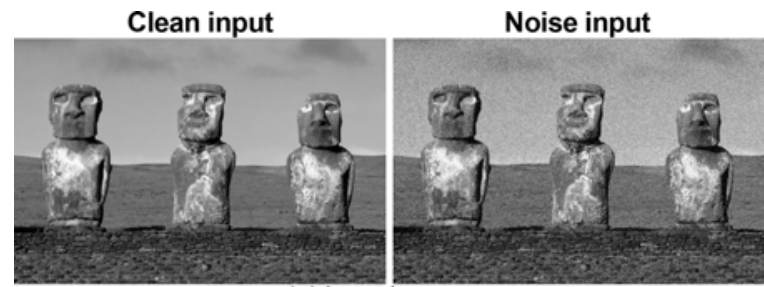

(a) input images

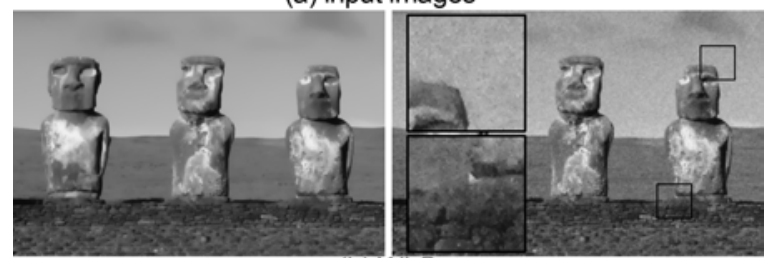

(b) WLS

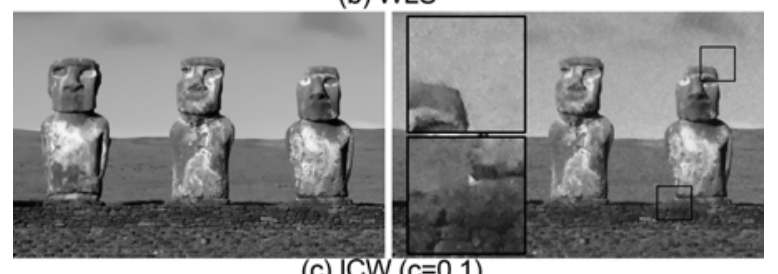

(c) ICW (c=0.1)

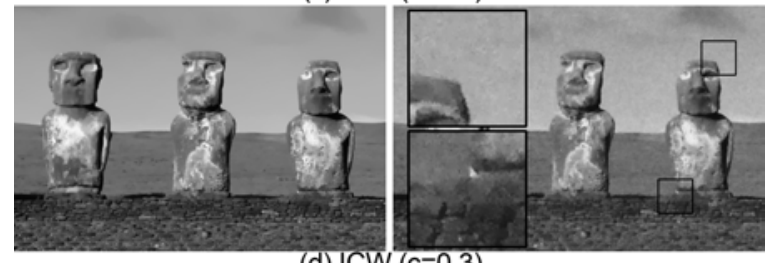

(d) ICW (c=0.3)

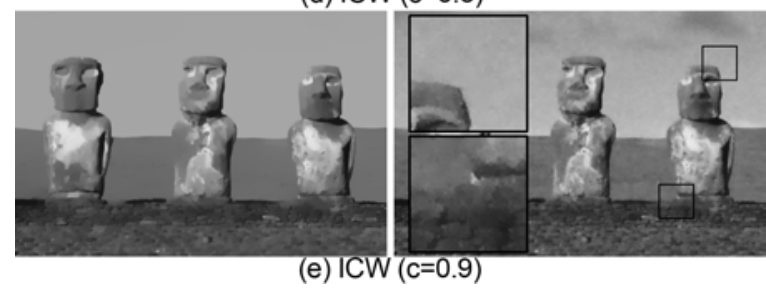

Fig. 1. The behavior of different noise power coefficient $(c)$ on a clean image (left column) and a noise image (right col$\mathrm{umn}$ ). The noise image is generated by adding a zero mean Gaussian noise with variance of 0.01 . A balanced noise power coefficient can reduce the noise effectively without degrading the clean image.

where $\Psi=D_{x}^{T} A_{x} D_{x}+D_{y}^{T} A_{y} D_{y}$, and $I_{m}$ denotes the identity matrix. The solution of this linear system is the same as the WLS-based decomposition, and therefore, it shares the similar frequency response [7]. As a result, we keep the same smoothing coefficient in our implementation.

The channel noise power $\left(\sigma_{c}^{2}\right)$ is a constant that controls the strength of noise reduction. It is selected from the vector $\vec{\Gamma}$ of all local variances $\left(\left\{\Gamma(p)=\sigma^{2}(p), p \in P\right\}\right)$ in ascending order. We name $c$ the noise power coefficient. And it indicates which value is selected from the vector. For example, $c=$ 0.5 indicates the median value of $\vec{\Gamma}$. In Equation (1), a small 
noise power coefficient indicates a small channel noise, and results a high fidelity between the base layer and the original image, as shown in Fig. 1 (c). If the noise power coefficient is too big, the base layer can be over smoothed, as shown in Fig. 1 (e), where the cloud is completely removed when there is no noise. A balanced noise power coefficient $(c=0.3$ is chosen in our implementation) ensures a good noise reduction result without over smoothing the clean image.
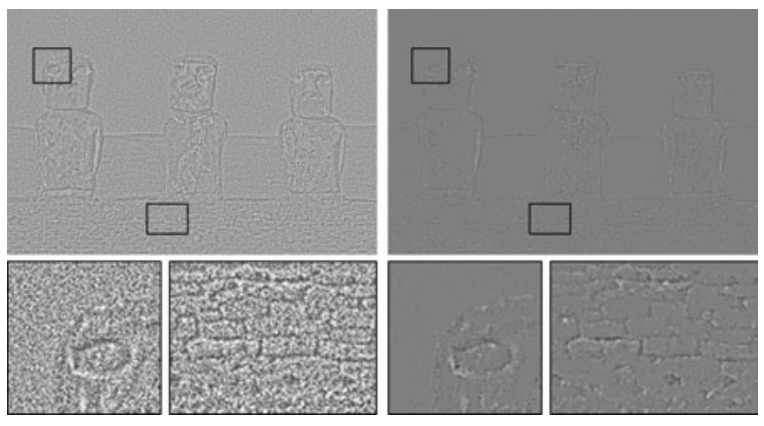

Fig. 2. Detail layer retrieved using (left) WLS-based decomposition, and (right) ICW-based decomposition. The input image is the same noise image presented in Fig. 1.

\subsection{Mutual Information-based Detail Substraction}

The detail layer is a reflectance component derived from the base layer and the original image luminance. Here, the noisereduced base layer is regarded as a clean reference. The information content is weighted as the amount of the mutual information between the clean reference and the original noise image as

$$
M(p)=\frac{1}{\alpha} \log _{2}\left(1+\beta \cdot\left(\frac{\sigma_{B \cdot I}(p)}{\sigma_{B}^{2}(p)}\right)^{\gamma}\right),
$$

where $\sigma_{B}^{2}(p)$ denotes the local variance of the base layer in a small window centred at $p, \sigma_{B \cdot I}(p)$ denotes the covariance between the base layer and the original luminance, $\beta$ and $\gamma$ are two constants control the effectiveness of the weighting function, and $\alpha$ is a normalization factor which fulfills the constraints of $M \in[0,1]$.

The mutual information ICW is a simplified information fidelity criteria [13], where $\sigma_{B \cdot I} / \sigma_{B}^{2}$ represents the signal attenuation caused by noise. As shown in Fig. 2, when generating detail layer, high weights are given to the pixels with more mutual information to the clean reference as

$$
D(p)=M(p) \cdot(I(p)-B(p)) .
$$

\section{EXPERIMENTAL RESULTS}

We first compare the proposed ICW-based tone mapping with the WLS-based tone mappingg [7] using the same parameters proposed in Farbman et al.'s original implementation.
Gaussian noise $(\mathrm{SNR}=15 \mathrm{~dB})$ was added in the clean HDR images, as shown in Fig. 3, where the reference image is generated from the clean HDR image using original WLS-based method. The proposed method improves the peak signal-tonoise ratio (PSNR) by $3 \mathrm{~dB}$ on the average, and improves the structure similarity index (SSIM) by $10-30 \%$, as shown in Table 1. More tests have been conducted at different noise levels, and the proposed ICWs improve the WLS-based tone mapping method by $1-4 \mathrm{~dB}$.

Table 1. Comparison of ICW to WLS [7].

\begin{tabular}{l|l|l|l}
\hline \hline Image & Quality metrics & WLS & ICW \\
\hline \multirow{2}{*}{ (a) Lamp } & PSNR (dB) & 30.08 & 33.26 \\
& SSIM & 0.6392 & 0.9113 \\
\hline \multirow{2}{*}{ (b) Memorial } & PSNR (dB) & 24.62 & 27.92 \\
& SSIM & 0.5601 & 0.7649 \\
\hline \multirow{2}{*}{ (c) Leaves } & PSNR (dB) & 26.43 & 28.10 \\
& SSIM & 0.7120 & 0.8186 \\
\hline \multirow{2}{*}{ (d) Desk } & PSNR (dB) & 30.49 & 34.74 \\
& SSIM & 0.6884 & 0.9398 \\
\hline
\end{tabular}

Visual comparisons are made on camera captured noise HDR images, as shown in Fig. 4, where the noise is significantly reduced. In our implementation, the $\mathrm{R}, \mathrm{G}, \mathrm{B}$ color channels are processed separately. This is due to lack of HDR color model, and may cause color shift in some pixels. The process can be improved when a more accurate HDR color model is found.

Five most representative tone mapping algorithms are chosen to compare with the proposed method: a global tone mapping operator [16], a subbands-based scale decomposition [11], a bilateral-filtering-based decomposition [8], a direct luminance compression [6], and the edge-preserving WLS [7]. Except the global tone mapping operator, which is implemented in an open source project Luminance HDR, the implementation of the other methods are provided by their authors. As shown in Fig. 5, different tone mapping algorithms give different visual experiences, which is very subjective. However, it is very obvious that the proposed method generates a cleaner displayable image.

\section{CONCLUSION}

In this paper, we presented a noise reduced tone mapping method based on information content weights working on base layer and detail layer, respectively. The experiments show that the proposed method effectively reduces the noise compared to the state-of-the-art tone mapping algorithms. The proposed method is suitable to be used for noise reduction on conventional image too, by replacing the input image from an HDR image to a conventional image. 


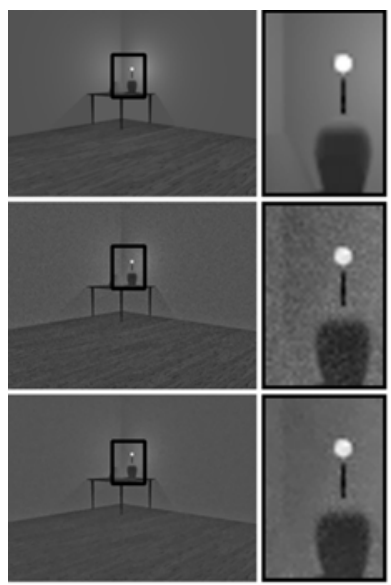

(a)

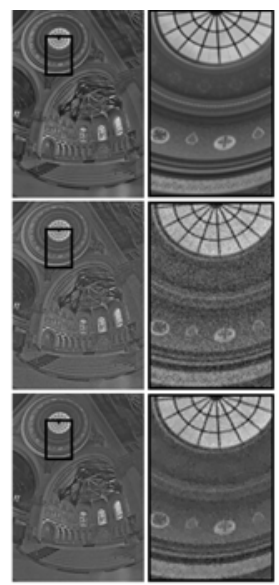

(b)

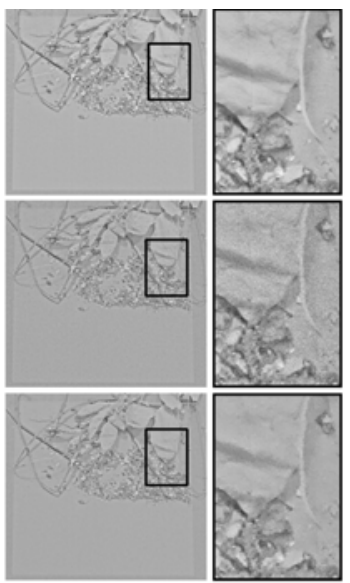

(c)

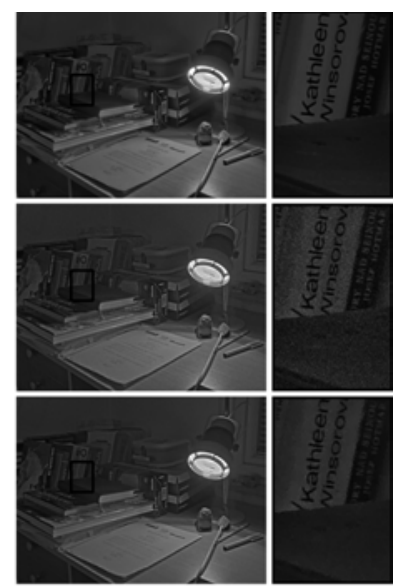

(d)

Fig. 3. Visual comparison of the luminance component generated using WLS (mid row) and ICW (bottom row). The clean image is specified in the top row.
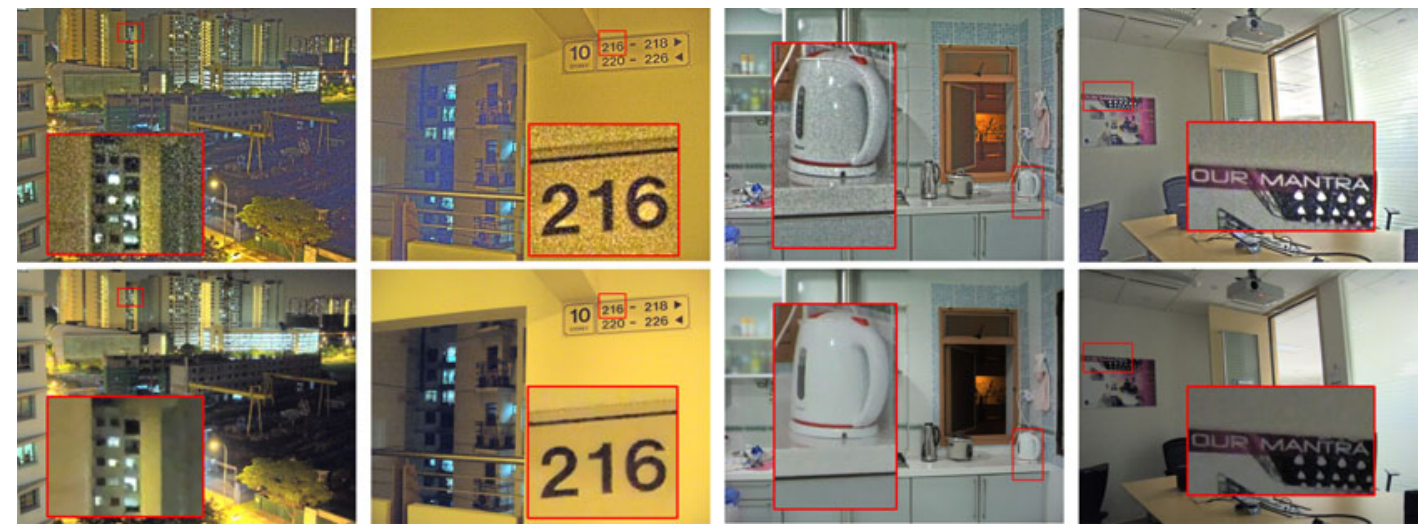

Fig. 4. Visual comparison of the color HDR image generated using WLS (top row) and ICW (bottom row).

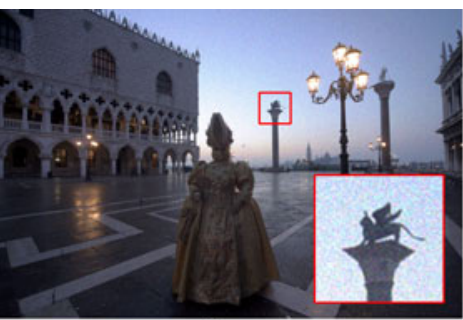

(a)

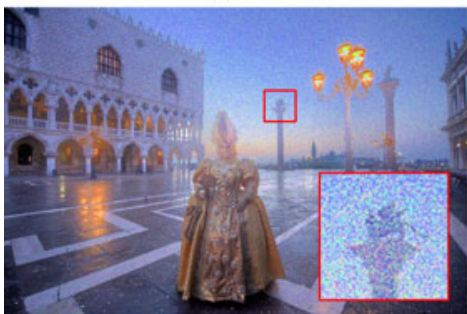

(d)

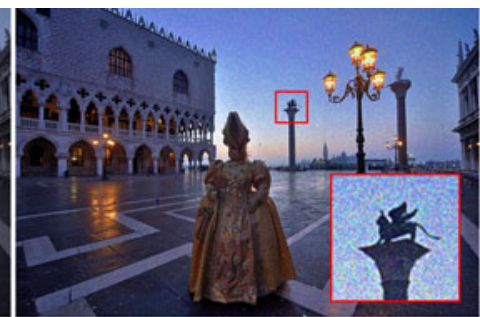

(b)

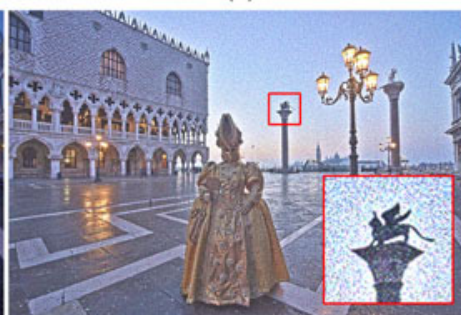

(e)

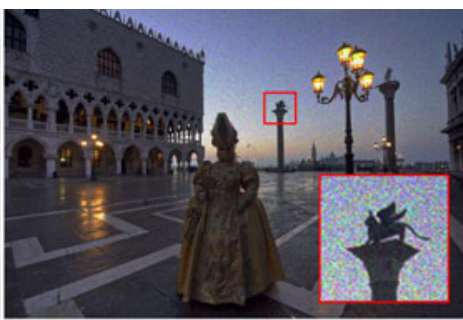

(c)

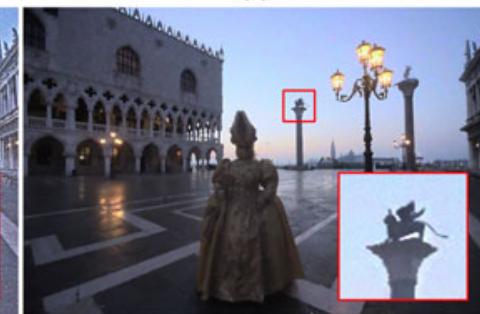

(f)

Fig. 5. Visual comparison of different tone mapping algorithms: (a) global tone curve [16]; (b) scale decomposition [11]; (c) bilateral filtering decomposition [8]; (d) direct luminance compression [6]; (e) WLS [7]; and (f) the proposed ICW. 


\section{REFERENCES}

[1] G. Wetzstein, I. Ihrke, and W. Heidrich, "Sensor saturation in fourier multiplexed imaging," in IEEE Conference on Computer Vision and Pattern Recognition (CVPR), 2010, pp. 545-552.

[2] P. E. Debevec and J. Malik, "Recovering high dynamic range radiance maps from photographs," in Proceedings of the 24th annual conference on Computer graphics and interactive techniques, ser. SIGGRAPH '97, 1997, pp. 369-378.

[3] H. Seetzen, W. Heidrich, W. Stuerzlinger, G. Ward, L. Whitehead, M. Trentacoste, A. Ghosh, and A. Vorozcovs, "High dynamic range display systems," ACM Trans. Graph., vol. 23, no. 3, pp. 760-768, Aug. 2004.

[4] R. Hoskinson, B. Stoeber, W. Heidrich, and S. Fels, "Light reallocation for high contrast projection using an analog micromirror array," ACM Trans. Graph., vol. 29, no. 6, pp. 165:1-165:10, Dec. 2010.

[5] G. Ward, "A contrast-based scalefactor for luminance display," in Graphics gems IV, 1994, pp. 415-421.

[6] Q. Shan, J. Jia, and M. Brown, "Globally optimized linear windowed tone mapping," IEEE Transactions on Visualization and Computer Graphics, vol. 16, no. 4, pp. 663-675, 2010.

[7] Z. Farbman, R. Fattal, D. Lischinski, and R. Szeliski, "Edge-preserving decompositions for multi-scale tone and detail manipulation," ACM Trans. Graph., vol. 27, no. 3, pp. 67:1-67:10, Aug. 2008.

[8] J. Kuang, G. M. Johnson, and M. D. Fairchild, "icam06: A refined image appearance model for hdr image rendering," J. Vis. Comun. Image Represent., vol. 18, no. 5, pp. 406-414, Oct. 2007.

[9] Z. Li, S. Rahardja, S. Yao, J. Zheng, and W. Yao, "High dynamic range compression by half quadratic regularization," in IEEE International Conference on Image Processing (ICIP), 2009, pp. 3169-3172.

[10] R. Fattal, D. Lischinski, and M. Werman, "Gradient domain high dynamic range compression," ACM Trans. Graph., vol. 21, no. 3, pp. 249-256, Jul. 2002.

[11] Y. Li, L. Sharan, and E. H. Adelson, "Compressing and companding high dynamic range images with subband architectures," ACM Trans. Graph., vol. 24, no. 3, pp. 836-844, Jul. 2005.

[12] J. W. Lee, R.-H. Park, and S. Chang, "Noise reduction and adaptive contrast enhancement for local tone mapping," IEEE Transactions on Consumer Electronics, vol. 58, no. 2, pp. 578-586, 2012.
[13] H. Sheikh, A. Bovik, and G. De Veciana, "An information fidelity criterion for image quality assessment using natural scene statistics," IEEE Transactions on Image Processing, vol. 14, no. 12, pp. 2117-2128, 2005.

[14] E. H. Land and J. J. McCann, "Lightness and retinex theory," J. Opt. Soc. Am., vol. 61, no. 1, pp. 1-11, Jan 1971.

[15] Z. Wang and X. Shang, "Spatial pooling strategies for perceptual image quality assessment," in IEEE International Conference on Image Processing (ICIP), 2006, pp. 2945-2948.

[16] R. Mantiuk, S. Daly, and L. Kerofsky, "Display adaptive tone mapping," ACM Trans. Graph., vol. 27, no. 3, pp. 68:1-68:10, Aug. 2008. 